\title{
Diameter-preserving maps on various classes of function spaces
}

\author{
by \\ Bruce A. Barnes (Eugene, OR) and Ashoke K. Roy (Calcutta)
}

\begin{abstract}
Under some mild assumptions, non-linear diameter-preserving bijections between (vector-valued) function spaces are characterized with the help of a well-known theorem of Ulam and Mazur. A necessary and sufficient condition for the existence of a diameter-preserving bijection between function spaces in the complex scalar case is derived, and a complete description of such maps is given in several important cases.
\end{abstract}

Introduction. Several papers on diameter-preserving linear bijections of function spaces have appeared in recent years (see, for example, [GM], $[\mathrm{GU}],[\mathrm{S}],[\mathrm{RR}])$. The present paper is a contribution to this circle of ideas, its principal motivation coming from an attempt to clarify and extend some of the results in $[R R]$ in response to an interesting question raised there.

To give a summary in this introduction to our main results, let us first explain briefly the notations and terminology that we use (unexplained terms and notations will be found in $[\mathrm{RR}]) . K, L$, and $S$ will generally denote compact convex sets in a Hausdorff locally convex topological vector space $X$ over $\mathbb{C}$ or $\mathbb{R}$, as the context will make clear. The (non-empty) set of extreme points of $K$ will be denoted by $\operatorname{ext}(K)$, and $A(K)$ will stand for the space of complex-valued continuous affine functions on $K$ endowed with the sup-norm. Our general references for facts concerning compact convex sets are $[\mathrm{A}]$ and $[\mathrm{AE}]$.

Let $Q$ be a compact Hausdorff space, and $X$ a Banach space. Then the diameter of $f \in C(Q, X)$ is defined by $d(f)=\sup _{x, y \in Q}\|f(x)-f(y)\|$. If $A_{1} \subseteq C\left(Q_{1}, X_{1}\right)$ and $A_{2} \subseteq C\left(Q_{2}, X_{2}\right)$ are function spaces, i.e. sup-norm closed subspaces, containing the constant functions, and separating points, then a (not necessarily linear) map $T: A_{1} \rightarrow A_{2}$ is called a diameterpreserving (d-preserving) bijection if $T$ is a 1-1 map of $A_{1}$ onto $A_{2}$, and $d(a)=d(T a)$ for all $a \in A_{1}$.

2000 Mathematics Subject Classification: Primary 46E25, 46A55, 47B38, 52A41. 
We begin, in Section 1, by discussing non-linear d-preserving bijections between (vector-valued) function spaces $A_{1}$ and $A_{2}$ of the kind described in the last paragraph and show, with the help of a well-known theorem of Mazur and Ulam (see [MU] and [B]), that under certain mild assumptions, $T$ can be characterized as $\bar{T}+\varphi$ where $\bar{T}: A_{1} \rightarrow A_{2}$ is a linear d-preserving bijection, and $\varphi: A_{1} \rightarrow X_{2}$ is a function, generally non-linear, with welldefined properties. We give several examples of such maps in Section 2.

In [RR, Theorem 1, p. 5], it was found that all linear d-preserving bijections between the spaces $A(K)$ and $A(L)$ are essentially induced by affine homeomorphisms between $K$ and $L$ when $K, L$ are compact convex sets with all points of $\operatorname{ext}(K)$ and $\operatorname{ext}(L)$ split. It was asked there whether the same conclusion could be derived by assuming the "splittability" property only for $\operatorname{ext}(K)$. We exhibit in Section 3 a very simple two-dimensional counterexample to this question involving a simplex $K$ and a hexagon $S$ for which a linear d-preserving bijection exists between $A_{\mathbb{R}}(K)$ and $A_{\mathbb{R}}(S)$. [Here the subscript $\mathbb{R}$ signifies real-valued functions.] The problem therefore naturally arises of characterizing compact convex sets $K_{1}$ and $K_{2}$ for which linear d-preserving bijections exist between $A\left(K_{1}\right)$ and $A\left(K_{2}\right)$. We consider the question more generally for function spaces $A_{i} \subseteq C\left(Q_{i}\right), i=1,2$, and show that the existence of an affine homeomorphism between certain compact convex sets in $A_{1}^{*}$ and $A_{2}^{*}$ - these sets being associated in a very natural manner with the state spaces of $A_{1}$ and $A_{2}$-is necessary and sufficient for this purpose. (For example, for the spaces $A_{\mathbb{R}}(K)$ and $A_{\mathbb{R}}(S)$ as above, the condition is that $K-K$ must be affinely homeomorphic to $S-S$.) We pursue this further by describing in Theorem 9 (in Section 4) the precise form of these d-preserving bijections from $A(K)$ to $A(S)$ where all the points in $\operatorname{ext}(K)$ are split and $S$ is any compact convex set when the aforementioned condition is satisfied.

The discussion in Section 3 leads naturally to the question of solvability in $S$ (a compact convex set) of the equation $S-S=K-K$ where $K$ is a given compact convex set with all points of $\operatorname{ext}(K)$ split. The question in general appears too difficult, but we can exhibit, in $\mathbb{R}^{n}$, the class of polytopes $S$ for which $S-S=K-K$ when $K$ is a given simplex in $\mathbb{R}^{n}$. This is the content of Section 5 .

1. d-Preserving maps on function spaces. Assume that $E$ is a normed linear space. Let $C(X, E)$ be the space of all continuous $E$-valued functions on $X$. For $v \in E$, we also denote by $v$ the constant function, $v(x)=v$ for all $x \in X$. Thus, vectors and constant functions are denoted in the same way; the meaning can be determined from the context. Also, we use $E$ to denote the closed subspace of $C(X, E)$ consisting of all the constant functions. 
A subspace $A \subseteq C(X, E)$ is a (vector-valued) function space if $A$ is closed in the sup-norm, $E \subseteq A$, and $A$ separates points of $X$. For $a \in A$, let $[a]=a+E$ be the residue class of $a$ in the quotient space $A / E$. We remark that $A$ is linearly isomorphic to $(A / E) \oplus E$. For let $\psi: A \rightarrow(A / E) \oplus E$ be defined by $\psi(a)=[a] \oplus a\left(x_{0}\right)$ where $x_{0} \in X$ is fixed. Then $\psi$ is 1-1 since if $a, b \in A$ with $\psi(a)=\psi(b)$, then $[a]=[b]$, so $a$ and $b$ differ by a constant, and also $a\left(x_{0}\right)=b\left(x_{0}\right)$. Thus, $a=b$. The linear map $\psi$ maps $A$ onto $(A / E) \oplus E$, since given $[a] \oplus v$, setting $c=a+\left(v-a\left(x_{0}\right)\right)$, we have $\psi(c)=[a] \oplus v$.

The diameter of $a \in A$ is defined by $d(a) \equiv \sup _{x, y \in X}\|a(x)-a(y)\|$.

Note that $d(a)=0$ if, and only if, $a$ is a constant function. For $[a] \in A / E$, define $d[a]=d(a)$. Then $[a] \mapsto d[a]$ is a norm on $A / E$ which we call the d-norm.

Proposition 1. Assume that $A_{1} \subseteq C\left(X_{1}, E_{1}\right)$ and $A_{2} \subseteq C\left(X_{2}, E_{2}\right)$ are function spaces. Make the linear identifications $A_{1} \approx\left(A_{1} / E_{1} \oplus E_{1}\right.$ and $A_{2} \approx\left(A_{2} / E_{2}\right) \oplus E_{2}$ as above, using the maps $\psi_{1}$ and $\psi_{2}, \psi_{k}\left(a_{k}\right)=\left[a_{k}\right] \oplus$ $a_{k}\left(x_{k}\right)$ where $x_{k} \in X_{k}$ are fixed points, $a_{k} \in A_{k}$ for $k=1,2$. Assume that $D: A_{1} / E_{1} \rightarrow A_{2} / E_{2}$ and $J: E_{1} \rightarrow E_{2}$ are linear bijections, and that $D$ is an isometry with respect to the d-norms. Define $D \oplus J:\left(A_{1} / E_{1}\right) \oplus E_{1} \rightarrow$ $\left(A_{2} / E_{2}\right) \oplus E_{2}$ by $(D \oplus J)([a] \oplus v)=D[a] \oplus J(v)$. Finally, define $\bar{D}: A_{1} \rightarrow$ $A_{2}$ by $\bar{D}(a)=\psi_{2}^{-1}(D \oplus J) \psi_{1}(a)$. Then $\bar{D}$ is a linear bijection which is $d$ preserving and has the property that the induced map $\widetilde{\bar{D}}: A_{1} / E_{1} \rightarrow A_{2} / E_{2}$ defined by $\widetilde{\bar{D}}([a])=[\bar{D}(a)]$ is $D$.

Proof. That $\bar{D}(a)$ is a linear bijection is clear, since $\psi_{2}^{-1}, D \oplus J$ and $\psi_{1}$ are all linear bijections. Let $a \in A_{1}$. Then by a straightforward computation,

$$
\bar{D}(a)=b+-b\left(x_{2}\right)+J\left(a\left(x_{1}\right)\right) \quad \text { where }[b]=D[a] .
$$

Thus, $d(\bar{D}(a))=d(b)=d[b]=d[D[a]]=d[a]=d(a)$, so $\bar{D}$ is d-preserving.

Remark. When $J: E_{1} \rightarrow E_{2}$ is a bijection, but not linear, and $D \oplus J$ and $\bar{D}$ are defined as above, then $\bar{D}$ is still a bijection and a d-preserving function which is not linear.

Let $A_{1}$ and $A_{2}$ be function spaces as above, and assume that $T: A_{1} \rightarrow A_{2}$ is a function (not necessarily linear). Now we prove a result which shows that with fairly weak assumptions on $T$ plus the assumption that $E_{1}$ and $E_{2}$ are linearly isomorphic, there exists a linear bijection $\bar{T}: A_{1} \rightarrow A_{2}$ with $\bar{T}$ d-preserving. The key tool here is the Ulam-Mazur Theorem [MU, B] which we state for the convenience of the reader: Assume that $\left(E_{1},\|\|_{1}\right)$ and $\left(E_{2},\|\|_{2}\right)$ are normed linear spaces and that $D: E_{1} \rightarrow E_{2}$ is a bijection with the properties: (a) $\|D(x)-D(y)\|_{2}=\|x-y\|_{1}$ for all $x, y \in E_{1}$, (b) $D(0)=0$, and in the case of complex scalars, (c) $D(i a)=i D(a)$ for all $a \in E_{1}$. Then $D$ is linear. 
Theorem 2. Assume that $T: A_{1} \rightarrow A_{2}$ is a function with the properties:

(1) $T$ is a bijection;

(2) $T$ has the property $d(T(a)-T(b))=d(a-b)$ for all $a, b \in A_{1}$;

(3) $T(0)=0$;

(4) [in the case of complex scalars] $T(i a)=i T(a)$ for all $a \in A_{1}$.

Also, assume that there exists $J: E_{1} \rightarrow E_{2}$ such that $J$ is a linear bijection. Then $T(a)=\bar{T}(a)+\varphi(a)$ where $\bar{T}: A_{1} \rightarrow A_{2}$ is a d-preserving linear bijection with $\bar{T}(v)=J(v), v \in E_{1}$, and $\varphi: A_{1} \rightarrow E_{2}$ is a function with the properties:

(i) $\varphi(0)=0$;

(ii) [in the complex scalar case $]$ for all $a \in A, \varphi(i a)=i \varphi(a)$;

(iii) the map $a \mapsto a+J^{-1}(\varphi(a))$ from $A_{1}$ to $A_{1}$ is $1-1$;

(iv) given $a \in A_{1}$, the equation $J(x)+\varphi(a+x)=0$ is solvable for $x \in E_{1}$.

Conversely, assume $\bar{T}: A_{1} \rightarrow A_{2}$ is a d-preserving linear bijection, and define $J: E_{1} \rightarrow E_{2}$ by $J(v)=\bar{T}(v), v \in E_{1}$. Further, assume that $\varphi: A_{1} \rightarrow$ $E_{2}$ is a function with properties (i)-(iv) above. Then $T(a)=\bar{T}(a)+\varphi(a)$ is a (possibly non-linear) map from $A_{1}$ onto $A_{2}$ having the properties (1)-(4) listed in the theorem.

Proof. Assume that $T: A_{1} \rightarrow A_{2}$ has properties (1)-(4). Define $\widetilde{T}$ : $A_{1} / E_{1} \rightarrow A_{2} / E_{2}$ in the obvious way: $\widetilde{T}([a])=[T a]$. From properties (1) and (2), $\widetilde{T}$ is a bijection and has property (a) above (in the statement of the Ulam-Mazur Theorem). Also, $\widetilde{T}$ inherits properties (3) and (4). Therefore the Ulam-Mazur Theorem applies, so $\widetilde{T}$ is linear. Now using the construction in Proposition 1, define $\bar{T}(a)=\psi_{2}^{-1}(\widetilde{T} \oplus J) \psi_{1}(a)$. By Proposition $1, \bar{T}$ is a linear bijection which is d-preserving. For $a \in A_{1}, \bar{T}(a)=T(a)+c$ for some $c \in E_{2}$. Define $\varphi(a)=T(a)-\bar{T}(a) \in E_{2}$. Thus, for all $a \in A_{1}$, $T(a)=\bar{T}(a)+\varphi(a)$. It follows from the definition of $\bar{T}$ that $\bar{T}(a)=J(a)$ when $a \in E_{1}$. That $\varphi$ satisfies (i) and (ii) is clear. We verify that (iii) and (iv) hold:

(iii) The map $a \mapsto a+J^{-1}(\varphi(a))$ from $A_{1}$ to $A_{1}$ is 1-1: Suppose $a+$ $J^{-1}(\varphi(a))=b+J^{-1}(\varphi(b))$. Then $v=b-a \in E_{1}$, and $v=J^{-1}(\varphi(a)-\varphi(b))$. Thus, $\bar{T}(v)=J(v)=\varphi(a)-\varphi(b)$. Therefore, $\bar{T}(b)-\bar{T}(a)=\varphi(a)-\varphi(b)$, so $T(a)=T(b)$. Then since $T$ is $1-1, a=b$.

(iv) Given $a \in A_{1}$, the equation $J(x)+\varphi(a+x)=0$ is solvable for $x \in E_{1}$ : Choose $b$ such that $T(b)=\bar{T}(a)$ ( $T$ is surjective). Then $0=d(T(a)-T(b))=$ $d(a-b)$, so $b=a+v$ for some $v \in E_{1}$. Thus, $\bar{T}(a)=T(b)=T(a+v)=$ $\bar{T}(a+v)+\varphi(a+v)=\bar{T}(a)+\bar{T}(v)+\varphi(a+v)=\bar{T}(a)+J(v)+\varphi(a+v)$. Therefore, $J(v)+\varphi(a+v)=0$.

Now we do the converse. Assume that $\bar{T}, J$, and $\varphi$ are as stated in the last paragraph of the theorem. Also, define $T: A_{1} \rightarrow A_{1}$ as above: $T(a)=$ 
$\bar{T}(a)+\varphi(a)$. That $T$ has properties (2), (3), and (4) follows immediately. We prove that $T$ is bijective.

$T$ is 1-1: Assume $T(a)=T(b)$, so

$$
\bar{T}(a)+\varphi(a)=\bar{T}(b)+\varphi(b) .
$$

Then $\bar{T}(a-b)=\varphi(b)-\varphi(a)$. Therefore, $a-b=x \in E_{1}$. Then $\bar{T}(a)=$ $\bar{T}(b)+J(x)$ (since $J=\bar{T}$ on $E_{1}$ ). Substituting this equality into (1), we have $\bar{T}(b)+J(x)+\varphi(a)=\bar{T}(b)+\varphi(b)$, so $J(x)=\varphi(b)-\varphi(a)$. Thus, $J(x)+\varphi(a)=\varphi(b)=\varphi(a-x)$. Then $a=b+x=b+J^{-1}(\varphi(b)-\varphi(a))$. Finally, this implies $a+J^{-1}(\varphi(a))=b+J^{-1}(\varphi(b))$. Applying (iii), it follows that $a=b$.

$T$ maps onto $A_{2}$ : Assume $c \in A_{2}$. We want to find $a \in A_{1}$ such that $\bar{T}(a)+\varphi(a)=c$. There exists $b \in A_{1}$ such that $c=\bar{T}(b)$. Now $\bar{T}(a)+\varphi(a)=$ $\bar{T}(b)$ implies $\bar{T}(b-a)=\varphi(a)$. Therefore, $b-a \in E_{1}$. Setting $x=b-a$, we have $\bar{T}(b)=\bar{T}(a)+J(x)$, so $\bar{T}(a)+\varphi(a)=\bar{T}(a)+J(x)$. Thus, $\varphi(a)=J(x)$. Then $\varphi(b-x)=J(x)$ and $J(-x)+\varphi(b-x)=0$. Finally, applying (iv), we can solve this last equation for $x$. Therefore, $a=b-x$ will be a solution of $T(a)=\bar{T}(a)+\varphi(a)=c$.

REMARK. $\bar{T}$, the linear part of $T$, has been characterized in [RR, Theorem 2 and Proposition 2] for some vector-valued function spaces.

The assumption in Theorem 2 of the existence of the linear bijection $J$ : $E_{1} \rightarrow E_{2}$ is not very restrictive. First, it is not assumed that $J$ is continuous, so the existence of $J$ is equivalent to $E_{1}$ and $E_{2}$ having (algebraic) bases of the same cardinality.

We use $\operatorname{card}(S)$ to denote the cardinality of a set $S$, and set $c=\operatorname{card}(\mathbb{R})$. Also, the dimension of a linear space $E$ is denoted by $\operatorname{dim}(E)$. Here is a useful known result:

$$
\text { If } \operatorname{dim}(E) \geq c \text {, then } \operatorname{card}(E)=\operatorname{dim}(E) \quad \text { [LT, Problem 2, p. 43]. }
$$

Note that because of the hypotheses in Theorem $2, T\left(E_{1}\right)=E_{2}$, and $T$ is 1-1. It follows that $\operatorname{card}\left(E_{1}\right)=\operatorname{card}\left(E_{2}\right)$. Thus, if $\operatorname{dim}\left(E_{1}\right) \geq c$ and $\operatorname{dim}\left(E_{2}\right) \geq c$, then

$$
\operatorname{dim}\left(E_{1}\right)=\operatorname{card}\left(E_{1}\right)=\operatorname{card}\left(E_{2}\right)=\operatorname{dim}\left(E_{2}\right) .
$$

Conclusion. If $\operatorname{dim}\left(E_{1}\right) \geq c$ and $\operatorname{dim}\left(E_{2}\right) \geq c$, then there exists a linear bijection of $E_{1}$ onto $E_{2}$.

Our main concern in this paper being with function spaces whose members take values in Banach spaces, it is pertinent to point out that, as a consequence of the Baire Category Theorem, the algebraic dimension of a Banach space is either finite or uncountable $(\geq c)$.

Now assume the dimensions of $E_{1}$ and $E_{2}$ are both finite, $E_{1} \approx \mathbb{R}^{m}$ and $E_{2} \approx \mathbb{R}^{n}$. Assume that $T$ and $T^{-1}$ are continuous, so that $T$ is a homeo- 
morphism of $E_{1} \approx \mathbb{R}^{m}$ onto $E_{2} \approx \mathbb{R}^{n}$. Then the Invariance of Dimension Theorem [D, p. 359] implies that $m=n$.

Conclusion. If $E_{1}$ and $E_{2}$ are both finite-dimensional and $T$ is a homeomorphism, then there exists a linear bijection of $E_{1}$ onto $E_{2}$.

2. Examples of non-linear d-preserving maps. In this section we present several examples of non-linear d-preserving maps $T$ which satisfy the hypotheses (1)-(4) of Theorem 2. Of course, by that theorem, $T$ must be the sum of a linear d-preserving bijection and a non-linear part.

First we give an example when $E_{1}=E_{2}=\mathbb{C}$. Let $A$ be a function space, $A \subseteq C(X)$. Note the relations for a function $a \in A: a=\operatorname{Re}(a)+i \operatorname{Im}(a)$; $i a=i \operatorname{Re}(a)-\operatorname{Im}(a) ; \operatorname{Re}(i a)=-\operatorname{Im}(a) ; \operatorname{Im}(i a)=\operatorname{Re}(a)$. Now set

$$
\varphi(a)=\sup (\operatorname{Re}(a))+i \sup (\operatorname{Im}(a))-\sup (-\operatorname{Re}(a))-i \sup (-\operatorname{Im}(a)) .
$$

For $a \in A, \varphi(i a)=i \varphi(a)$. Proof:

$$
\begin{aligned}
\varphi(i a) & =\sup (\operatorname{Re}(i a))+i \sup (\operatorname{Im}(i a))-\sup (-\operatorname{Re}(i a))-i \sup (-\operatorname{Im}(i a)) \\
& =\sup (-\operatorname{Im}(a))+i \sup (\operatorname{Re}(a))-\sup (\operatorname{Im}(a))-i \sup (-\operatorname{Re}(a)) \\
& =i[\sup (\operatorname{Re}(a))-\sup (-\operatorname{Re}(a))+i \sup (\operatorname{Im}(a))-i \sup (-\operatorname{Im}(a))] \\
& =i \varphi(a) .
\end{aligned}
$$

Note. For $b \in A, \mu \in \mathbb{C}, \varphi(b+\mu)=\varphi(b)+2 \mu$. Proof:

$$
\begin{aligned}
\varphi(b+\mu)= & {[\sup (\operatorname{Re}(b))+\operatorname{Re}(\mu)]+i[\sup (\operatorname{Im}(b))+\operatorname{Im}(\mu)] } \\
& -[\sup (-\operatorname{Re}(b))-\operatorname{Re}(\mu)]-i[\sup (-\operatorname{Im}(b))-\operatorname{Im}(\mu)] \\
= & \varphi(b)+2 \mu .
\end{aligned}
$$

Now define $T(a)=a+\varphi(a)$. Then $T$ maps onto $A$. Proof: Given $b \in A$, we want $a \in A$ such that $b=a+\varphi(a)$. Set $a=b+\mu$ where $\mu \in \mathbb{C}$. Then we want that $b=(b+\mu)+\varphi(b+\mu)=b+\mu+\varphi(b)+2 \mu$ (from Note). Solving, we see that $3 \mu=-\varphi(b)$. Letting $\mu=-\frac{1}{3} \varphi(b)$, we find that $T(a)=b$.

$T$ is 1-1. Proof: Suppose that $a, b \in A$ with $a+\varphi(a)=b+\varphi(b)$, so $a-b=\varphi(b)-\varphi(a)$. Then $a=b+\mu$ where $\mu \in \mathbb{C}$. Therefore, $\mu=\varphi(b)-\varphi(a)$ $=\varphi(a-\mu)-\varphi(a)=($ from Note $) \varphi(a)-2 \mu-\varphi(a)=-2 \mu$, so $\mu=0$.

Thus, $T(a)=a+\varphi(a)$ is non-linear, but satisfies the hypotheses of Theorem 2.

When the scalar field is $\mathbb{R}$, there is a much simpler example. Assume that $A$ is a function space of $\mathbb{R}$-valued functions. The reader can check that $T(a)=a+\sup (a)$ or $T(a)=a+\max [\sup (a), 0], a \in A$, is a non-linear bijection of $A$ onto $A$ that satisfies the hypotheses of Theorem 2 . The latter definition of $T$ shows that the function $\varphi(x)$ in the statement of Theorem 2 is not generally surjective. 
Now we give an example in the vector-valued case. Let $Y$ be a compact Hausdorff space, and set $E_{1}=E_{2}=C(Y)$. We construct a function $J$ with the properties:

(i) $J$ is a bijection of $C(Y)$ onto $C(Y)$;

(ii) $J(0)=0$ and $J(i f)=i J(f)$ for all $f \in C(Y)$;

(iii) $J$ is not linear.

For $f \in C(Y)$, define

$$
J(f)=[\operatorname{Re}(f)]^{3}-[\operatorname{Im}(f)]^{3}-i[\operatorname{Re}(i f)]^{3}+i[\operatorname{Im}(i f)]^{3} .
$$

[In the real scalar case, $J(f)=f^{3}$ will work.] (ii) follows from a straightforward computation, and (iii) is clear. We verify (i).

$J$ is 1-1. Proof: Suppose $f_{k} \in C(Y), f_{k}=u_{k}+i v_{k}$, where $u_{k}, v_{k}$ are $\mathbb{R}$-valued functions in $C(Y), k=1,2$. Note that $i f_{k}=-v_{k}+i u_{k}, k=1,2$. Suppose that $J\left(f_{1}\right)=J\left(f_{2}\right)$. Then $u_{1}^{3}-v_{1}^{3}=u_{2}^{3}-v_{2}^{3}$, and $u_{1}^{3}+v_{1}^{3}=u_{2}^{3}+v_{2}^{3}$. Therefore, $2 u_{1}^{3}=2 u_{2}^{3}$, so $u_{1}=u_{2}$. Then $v_{1}^{3}=v_{2}^{3}$, so $v_{1}=v_{2}$.

$J$ maps onto $C(Y)$. Proof: Assume that $g=u+i v \in C(Y), u, v \mathbb{R}$-valued. Set

$$
f=\left[\frac{u+v}{2}\right]^{1 / 3}+i\left[\frac{v-u}{2}\right]^{1 / 3}, \quad \text { so } \quad \text { if }=i\left[\frac{u+v}{2}\right]^{1 / 3}-\left[\frac{v-u}{2}\right]^{1 / 3} .
$$

Then

$$
J(f)=\left[\frac{u+v}{2}\right]-\left[\frac{v-u}{2}\right]+i\left[\frac{v-u}{2}\right]+i\left[\frac{u+v}{2}\right]=u+i v=g .
$$

Now let $A \subseteq C(X, C(Y))$ be a function space. Let $I$ denote the identity map on $A$. Use Proposition 1 to define $T=\psi^{-1}(I \oplus J) \psi$ where $\psi$ is the map defined in the discussion just prior to Proposition 1 . Then $T$ is a non-linear bijection with properties (1)-(4) in Theorem 2.

3. d-Preserving maps on complex-valued function spaces. The real-scalar version of Theorem 1 in $[\mathrm{RR}]$ is:

Let $K$ and $S$ be compact convex sets, both of which have the property that every extreme point is a split face. If $D: A(K) \rightarrow A(S)$ is a d-preserving linear bijection, then there exists an affine homeomorphism $\tau: S \rightarrow K$ and a functional $\alpha$ defined on $A(K)$ such that for all $a \in A(K)$,

$$
D(a)=c(a \circ \tau)+\alpha(a), \quad \text { where } c= \pm 1, \text { and } \alpha(1) \neq-c .
$$

A question raised in [RR, Remark 1] is: Does the same result hold if the hypothesis, "every extreme point is a split face", is assumed only for $K$ ? We now give an example which answers this question in the negative.

In $\mathbb{R}^{2}$, let $K=\operatorname{co}\{(1,0),(-1,0),(0,1)\}$. The three extreme points of $K$ are split faces in the sense defined in $[\mathrm{A}]$ because $K$ is a simplex, and for each extreme point $x$ of $K,\{x\}^{\prime}$ (三 the complementary set of $\{x\}$ ) is a face, 
and every point $p \in K$ can be written uniquely as $p=\alpha x+(1-\alpha) y$, where $y \in\{x\}^{\prime}$ and $0 \leq \alpha \leq 1$. [Strictly speaking, to use the definition given in [A], one should regard $K \subseteq\left\{\varphi \in A(K)^{*}: \varphi(1)=1\right\}$. The analysis could be done in $A(K)^{*}$. But this seems an unnecessary and technical approach to the elementary example under consideration.]

Now let $S=\frac{1}{2}(K-K)$. It is easily checked that $S$ is a hexagon; in fact $S=\operatorname{co}\left\{x_{1}, x_{2}, x_{3},-x_{1},-x_{2,},-x_{3}\right\}$ where $x_{1}=(1,0), x_{2}=(1 / 2,1 / 2)$, and $x_{3}=(-1 / 2,1 / 2)$.

Thus, there is no affine homeomorphism of $S$ onto $K$. Using the identity

$$
\frac{1}{2}(z-w)-\frac{1}{2}(x-y)=\left(\frac{z+y}{2}\right)-\left(\frac{w+x}{2}\right),
$$

it is easy to check that $K-K=S-S$. Then it follows from Corollary 7 of this paper that there exists a d-preserving linear bijection of $A(K)$ onto $A(S)$.

Now we investigate function spaces $A_{i} \subseteq C\left(Q_{i}\right), i=1,2$, to determine necessary and sufficient conditions for the existence of a linear d-preserving $T: A_{1} \rightarrow A_{2}$. First we give some relevant definitions and results which will be needed for this analysis.

Let $S$ be a compact convex set which is symmetric $(s \in S \Rightarrow-s \in S)$. When the scalar field is $\mathbb{R}$, define $A_{0}(S)=\{f \in A(S): f(0)=0\}$. When the scalar field is $\mathbb{C}$, assume $(s \in S, \alpha \in \mathbb{C},|\alpha|=1) \Rightarrow \alpha s \in S$. In this case define $A_{0}(S)=\{f \in A(S): f(i s)=i f(s)$ for all $s \in S\}$. Note that if $f \in A_{0}(S)$, then $f(i 0)=i f(0)$, so $f(0)=0$.

Proposition 3. Let $X$ be a Banach space. Assume that $f \in A_{0}\left(X_{1}^{*}\right)$ (here $X_{1}^{*}$ is the closed unit ball of the dual of $X$, and the topology on $X_{1}^{*}$ is the $w^{*}$-topology). Extend $f$ to $\tilde{f}$ by

$$
\widetilde{f}(\varphi)=\|\varphi\| f(\varphi /\|\varphi\|), \quad \varphi \in X^{*} .
$$

Then $\widetilde{f}$ is a $w^{*}$-continuous linear functional on $X^{*}$.

Proof. We do the complex scalar case, so it is assumed that $f(i \varphi)=$ if $(\varphi)$ for all $\varphi \in X_{1}^{*}$. Also, note that $f$ has the properties: $f(-\varphi)=-f(\varphi)$; and $\left(0 \leq \alpha \leq 1, \varphi \in X_{1}^{*}\right) \Rightarrow \alpha f(\varphi)=f(\alpha \varphi)$. To be proved:

(a) $\widetilde{f}(\varphi+\psi)=\widetilde{f}(\varphi)+\widetilde{f}(\psi)$ for all $\varphi, \psi \in X^{*}$;

(b) $\widetilde{f}(\alpha \varphi)=\alpha \widetilde{f}(\varphi)$ for all $\alpha \in \mathbb{C}, \varphi \in X^{*}$.

Assume that both (a) and (b) hold. Now $\operatorname{ker}(\widetilde{f}) \cap X_{1}^{*}=\{$ the zero set of $f\}$, which is $\mathrm{w}^{*}$-closed. Therefore by the Krĕn-Shmul'yan Theorem (see [DS] or [LT]), $\tilde{f}$ is $\mathrm{w}^{*}$-continuous. 
Now we prove (a). Let $\varphi, \psi \in X^{*} \backslash\{0\}$. Since $\|\varphi+\psi\| /(\|\varphi\|+\|\psi\|) \leq 1$,

$$
\left(\frac{\|\varphi+\psi\|}{\|\varphi\|+\|\psi\|}\right) f\left(\frac{\varphi+\psi}{\|\varphi+\psi\|}\right)=f\left(\frac{\varphi+\psi}{\|\varphi\|+\|\psi\|}\right) .
$$

Therefore

$$
\begin{aligned}
\widetilde{f}(\varphi+\psi) & =\|\varphi+\psi\| f\left(\frac{\varphi+\psi}{\|\varphi+\psi\|}\right)=(\|\varphi\|+\|\psi\|) f\left(\frac{\varphi+\psi}{\|\varphi\|+\|\psi\|}\right) \\
& =(\|\varphi\|+\|\psi\|)\left[\frac{\|\varphi\|}{\|\varphi\|+\|\psi\|} f\left(\frac{\varphi}{\|\varphi\|}\right)+\frac{\|\psi\|}{\|\varphi\|+\|\psi\|} f\left(\frac{\psi}{\|\psi\|}\right)\right] \\
& =\|\varphi\| f\left(\frac{\varphi}{\|\varphi\|}\right)+\|\psi\| f\left(\frac{\psi}{\|\psi\|}\right)=\widetilde{f}(\varphi)+\widetilde{f}(\psi) .
\end{aligned}
$$

To prove (b), first note that

$$
\widetilde{f}(-\varphi)=\|\varphi\| f\left(\frac{-\varphi}{\|\varphi\|}\right)=-\|\varphi\| f\left(\frac{\varphi}{\|\varphi\|}\right)=-\widetilde{f}(\varphi)
$$

Now suppose $\alpha \in \mathbb{R}$ and $\alpha>0$. Then $\tilde{f}(\alpha \varphi)=\|\alpha \varphi\| f(\alpha \varphi /\|\alpha \varphi\|)=$ $\alpha\|\varphi\| f(\varphi /\|\varphi\|)=\alpha \widetilde{f}(\varphi)$. The same equality for $\alpha \in \mathbb{R}$ and $\alpha<0$ follows from this and the fact that $\widetilde{f}(-\varphi)=-\widetilde{f}(\varphi)$. Also, $\widetilde{f}(i \varphi)=\|i \varphi\| f(i \varphi /\|i \varphi\|)$ $=\|\varphi\| f(i \varphi /\|i \varphi\|)=i\|\varphi\| f(\varphi /\|\varphi\|)=i \widetilde{f}(\varphi)$.

Finally, assume that $\alpha=\beta+i \delta, \beta, \delta \in \mathbb{R}$. Then

$$
\widetilde{f}(\alpha \varphi)=\widetilde{f}(\beta \varphi+i \delta \varphi)=\widetilde{f}(\beta \varphi)+\widetilde{f}(i \delta \varphi)=\beta \widetilde{f}(\varphi)+i \delta \widetilde{f}(\varphi) .
$$

Let $A \subseteq C(Q)$ where $Q$ is a compact Hausdorff space, be a function space equipped with the usual sup-norm $\|a\|_{\infty}$. We work in the complex scalar case. For $[a] \in A / \mathbb{C}$, define

$$
\|[a]\|_{\infty}=\inf \left\{\|a+\lambda\|_{\infty}: \lambda \in \mathbb{C}\right\},
$$

the usual quotient norm.

Note 4. The d-norm on $A / \mathbb{C}$ is equivalent to the quotient norm.

Proof. For $a \in A$, fix $x, y \in X$ such that $d(a)=|a(x)-a(y)|$. Note that it is clear that $d(a) \leq 2\|a\|_{\infty}$, so for all $\lambda \in \mathbb{C}, d(a)=d(a+\lambda) \leq 2\|a+\lambda\|_{\infty}$. It follows that $d[a]=d(a) \leq 2\|[a]\|_{\infty}$. Also, $\mid\|[a]\|_{\infty} \leq\|a-a(y)\|_{\infty}=$ $\sup _{z \in X}|a(z)-a(y)|=|a(x)-a(y)|=d(a)=d[a]$.

Note that by the Hahn-Banach Theorem, $(A / \mathbb{C})^{*}$ is isometrically isomorphic to $\left\{\varphi \in A^{*}: \varphi(1)=0\right\}$. For $a \in A, \varphi \in(A / \mathbb{C})^{*}$, let $\widehat{[a]}(\varphi)=\varphi([a])$. Define $\Gamma=\{\alpha \in \mathbb{C}:|\alpha|=1\}$, and $T=\{\alpha(q-r): \alpha \in \Gamma, q, r \in Q\} \subseteq(A / \mathbb{C})^{*}$ (here we identify $Q$ as a subset of $A^{*}$ via the evaluation map $q \mapsto e_{q}$ where 
$e_{q} \in A^{*}$ is defined by $\left.e_{q}(a)=a(q), a \in A, q \in Q\right)$. Then

$$
\begin{aligned}
T^{0} & =\{[a]: \operatorname{Re}(\alpha(a(q)-a(r))) \leq 1 \text { for all } \alpha \in \Gamma, \text { and all } q, r \in Q\} \\
& =\{[a]: d[a]=d(a) \leq 1\} \\
& =\{\text { the closed unit ball in } A / \mathbb{C} \text { with respect to the d-norm }\} . \\
T^{00} & =\left\{\varphi \in(A / \mathbb{C})^{*}: \operatorname{Re}(\widehat{[a]}(\varphi)) \leq 1 \text { for all }[a] \in T^{0}\right\} \\
& =\left\{\text { the closed unit ball in }(A / \mathbb{C})^{*} \text { with respect to the dual d-norm }\right\}, \\
& =\overline{\operatorname{co}}(T) \quad(\text { by the Bipolar Theorem }[\mathrm{LT}, \text { Thm. } 7.3, \text { p. } 162]) \\
& =\overline{\operatorname{co}}\left(\Gamma\left(S_{A}-S_{A}\right)\right)=\overline{\operatorname{aco}}\left(S_{A}-S_{A}\right)
\end{aligned}
$$

where $S_{A}=\left\{\varphi \in A^{*}:\|\varphi\|=\varphi(1)=1\right\}$ is the state space of $A$, and $\overline{\operatorname{aco}}(S)$ is the absolute convex hull of a set $S\left(\subseteq A^{*}\right)$ where the closure is taken with respect to the $\mathrm{w}^{*}$-topology in $A^{*}$.

We use this notation in the theorem below.

TheOREM 5. The map $[a] \mapsto \widehat{[a]}$ is a linear bijection of $A / \mathbb{C}$ onto $A_{0}\left(T^{00}\right)$. Also, it is an isometry of $(A / \mathbb{C}, d$-norm $)$ onto $\left(A_{0}\left(T^{00}\right),\|\|_{\infty}\right)$ where for $b \in A_{0}\left(T^{00}\right)$,

$$
\|b\|_{\infty}=\sup \left\{|b(\varphi)|: \varphi \in T^{00}\right\}
$$

Proof. Since $T^{00}=\left\{\right.$ the closed unit ball in $(A / \mathbb{C})^{*}$ with respect to the dual d-norm $\}$, for $a \in A, d[a]=\sup \left\{|\varphi(a)|: \varphi \in T^{00}\right\}=\|\widehat{[a]}\|_{\infty}$. Thus, the map $[a] \mapsto \widehat{[a]}$ is an isometry. This map is clearly linear and $1-1$. Now assume that $b \in A_{0}\left(T^{00}\right)$. Proposition 3 applies with $X=(A / \mathbb{C}, \mathrm{d}$-norm $)$. Therefore $b$ has a $\mathrm{w}^{*}$-continuous extension $\widetilde{b}$ in $(A \mathbb{C})^{* *}$. It follows that there exists $[a] \in A / \mathbb{C}$ such that $\widehat{[a]}=b$.

Let $J$ and $K$ be convex circled subsets of a complex linear space. We say that an affine map $\tau: J \rightarrow K$ is a complex affine map if $\tau(i x)=i \tau(x)$ for all $x \in J$. [Note that the map $z \mapsto \bar{z}$ on the closed unit disk in the complex plane is affine, but not complex affine.]

Now let $A_{k} \subseteq C\left(Q_{k}\right), k=1,2$, be function spaces on compact Hausdorff spaces $Q_{1}$ and $Q_{2}$. Let $D$ be a linear isometry of $\left(A_{1} / \mathbb{C}\right.$, d-norm) onto $\left(A_{2} / \mathbb{C}, \mathrm{d}\right.$-norm $)$. Letting $T_{k}=\left\{\alpha(q-r): \alpha \in \Gamma, q, r \in Q_{k}\right\}, k=1,2$, we deduce by the discussion prior to Theorem 5 that $T_{k}^{00}$ is the closed unit ball in $\left(A_{k} \mathbb{C}\right)^{*}$. Thus, $D^{*}\left(T_{2}^{00}\right)=T_{1}^{00}$, where $D^{*}$ is the adjoint of $D$. For $\varphi \in T_{2}^{00}$, define $\tau(\varphi)=D^{*}(\varphi)$. Then $\tau$ is a complex affine homeomorphism (w*-topology) of $T_{2}^{00}$ onto $T_{1}^{00}$. Also, for $\varphi \in T_{2}^{00},[a] \in A_{1} / \mathbb{C}$, $\widehat{D[a}](\varphi)=\widehat{[a]}\left(D^{*}(\varphi)\right)=\widehat{[a]}(\tau(\varphi))$, so $\widehat{D[a]}=\widehat{[a]} \circ \tau,[a] \in A_{1} / \mathbb{C}$.

We summarize this discussion in the following theorem. 
THEOREM 6. There exists a d-preserving linear bijection of $A_{1}$ onto $A_{2}$ if, and only if, there exists a complex affine homeomorphism of the set $\overline{\operatorname{aco}}\left(S_{A_{2}}-S_{A_{2}}\right)$ onto $\overline{\operatorname{aco}}\left(S_{A_{1}}-S_{A_{1}}\right)$.

Proof. First note that $T_{k}$ is compact as it is the continuous image of the compact set $\Gamma \times Q_{k} \times Q_{k}$ under the map $(\alpha, q, r) \mapsto \alpha(q-r)$. Thus, $T_{k}^{00}=\overline{\operatorname{aco}}\left(S_{A_{k}}-S_{A_{k}}\right)$ is compact. Suppose that $\tau: \overline{\operatorname{aco}}\left(S_{A_{2}}-S_{A_{2}}\right) \rightarrow$ $\overline{\operatorname{aco}}\left(S_{A_{1}}-S_{A_{1}}\right)$ is a complex affine homeomorphism. Then for $a \in A_{1}, D \widehat{[a]}=$ $\widehat{[a]} \circ \tau$ is a linear bijection of $A_{0}\left(T_{1}^{00}\right)$ onto $A_{0}\left(T_{2}^{00}\right)$ which is an isometry with respect to the sup-norm. By Theorem 5 , this implies the existence of a linear bijection $\widetilde{D}$ which is an isometry of $\left(A_{1} / \mathbb{C}, \mathrm{d}\right.$-norm $)$ onto $\left(A_{2} / \mathbb{C}, \mathrm{d}\right.$-norm $)$. Then by Proposition 1, $\widetilde{D}$ lifts to a linear bijection of $A_{1}$ onto $A_{2}$ which is d-preserving.

Conversely, assume that $\bar{D}$ is a linear bijection of $A_{1}$ onto $A_{2}$ which is d-preserving. Define $D: A_{1} \mathbb{C} \rightarrow A_{2} / \mathbb{C}$, as usual, by $D[a]=[\bar{D}(a)]$. Then $D$ is a linear bijection which is an isometry with respect to the d-norm. Then as argued in the discussion before the theorem, $D^{*}$ is a complex affine homeomorphism of $T_{2}^{00}=\overline{\operatorname{aco}}\left(S_{A_{2}}-S_{A_{2}}\right)$ onto $T_{1}^{00}=\overline{\operatorname{aco}}\left(S_{A_{1}}-S_{A_{1}}\right)$.

When $A_{k}$ is the space $A\left(K_{k}\right)$, i.e., the space of continuous affine functions on a compact convex set $K_{k}$ with the sup-norm, then $S_{A_{k}}=K_{k}, k=1,2$. In the real scalar case, we see that $T_{k}^{00}=\overline{\mathrm{co}}\left(K_{k}-K_{k}\right)=K_{k}-K_{k}$.

Also note that if $\tau: K_{2}-K_{2} \rightarrow K_{1}-K_{1}$ is an affine homeomorphism, then $\tau$ carries a point of symmetry to a point of symmetry, and 0 is the only point of symmetry for both the above sets. Therefore, we must have $\tau(0)=0$.

Using the same notation as in Theorem 6 , we have the following corollary:

COROllary 7 . In the case where the scalar field is $\mathbb{R}$, there exists a $d$-preserving linear bijection of $A\left(K_{1}\right)$ onto $A\left(K_{2}\right)$ if, and only if, there exists an affine homeomorphism of $K_{2}-K_{2}$ onto $K_{1}-K_{1}$.

The proof of Theorem 6 applies verbatim to Corollary 7, except that $T_{k}^{00}=K_{k}-K_{k}, k=1,2$, as we noted above.

Corollary 7 raises the natural question: When $K_{1}$ and $K_{2}$ are compact convex sets, under what conditions are $K_{1}-K_{1}$ and $K_{2}-K_{2}$ affinely homeomorphic? This question seems too difficult to answer in general, although in some cases conditions can be found. For example, the results in [RR] show that (in the real scalar case), when $K_{1}$ and $K_{2}$ both have the property that all their extreme points are split faces, then $K_{1}-K_{1}$ and $K_{2}-K_{2}$ are affinely homeomorphic if, and only if, $K_{1}$ and $K_{2}$ are affinely homeomorphic.

Here is an especially simple situation. Suppose that $K$ is a compact convex set which is symmetric. Then clearly $K+K=K-K$. Also, $K+K=$ $2 K$, since for all $x, y \in K, x+y=2\left(\frac{x+y}{2}\right)$. Thus, $K-K=2 K$. It follows that, 
when both $K_{1}$ and $K_{2}$ are symmetric, then again $K_{1}-K_{1}$ and $K_{2}-K_{2}$ are affinely homeomorphic if, and only if, $K_{1}$ and $K_{2}$ are affinely homeomorphic.

We derive more information concerning this question in the last section.

4. A characterization of some linear d-preserving maps. Assume that $L$ and $S$ are compact convex sets, that $0 \in L, 0 \in S$, and $\overline{\operatorname{aco}}(L-L)=$ $\overline{\mathrm{aco}}(S-S)$ [in the case of real scalars, the assumption is $L-L=S-S$ ]. For $a \in A(L)$, the function $\widehat{a}=\widehat{[a]}$ is in $A_{0}(\overline{\operatorname{aco}}(L-L))$. Now for $a \in A(L)$, define $a_{S} \in A(S)$ by $a_{S}=\left.\widehat{a}\right|_{S}+a(0)$ on $S$. Since the hypotheses are the same for $L$ and $S$, for $a \in A(S)$, we define $a_{L}$ in the same way. Note that $a_{S} \in A(S)$ and $a_{L} \in A(L)$. Also, $a_{S}(0)=a_{L}(0)=a(0)$. We use this notation in the next result.

Proposition 8. Assume that $L$ and $S$ are compact convex sets with the properties above.

(1) For $a \in A(L), \widehat{a}_{S}=\widehat{a}$; for $a \in A(S), \widehat{a}_{L}=\widehat{a}$.

(2) For $a \in A(L),\left(a_{S}\right)_{L}=a$; for $a \in A(S),\left(a_{L}\right)_{S}=a$.

(3) For $a \in A(L), d(a)=d\left(a_{S}\right)$; for $a \in A(S), d(a)=d\left(a_{L}\right)$.

Also, if $\lambda \in A(L)$ is a constant function, then $\lambda_{S}=\lambda$ (and the same statement with $L$ and $S$ interchanged).

Proof. We do the proof in the complex scalar case.

First we prove (1) when $a \in A(L)$. It is enough to verify that $\widehat{a}_{S}(\varphi)=$ $\widehat{a}(\varphi)$ for all $\varphi \in \overline{\operatorname{aco}}(S-S)$ of the form $\varphi=t\left(s_{1}-s_{2}\right),|t|=1, s_{1}, s_{2} \in S$, since these generate $\overline{\operatorname{aco}}(S-S)$. Assume that $\varphi=t\left(s_{1}-s_{2}\right)$ as above, and $a \in A(L)$. Now $\frac{1}{2}\left(\varphi+t s_{2}\right)=\frac{1}{2} t s_{1}$, so $\frac{1}{2} \widehat{a}(\varphi)+\frac{1}{2} t \widehat{a}\left(s_{2}\right)=\frac{1}{2} t \widehat{a}\left(s_{1}\right)$. Therefore, $\widehat{a}_{S}(\varphi)=t\left(a_{S}\left(s_{1}\right)-a_{S}\left(s_{2}\right)\right)=t\left(\widehat{a}\left(s_{1}\right)-\widehat{a}\left(s_{2}\right)\right)=\widehat{a}(\varphi)$.

Now we prove (2) for $a \in A(L)$. By definition $\left(a_{S}\right)_{L}=\left.\widehat{a}_{S}\right|_{L}+a_{S}(0)$, so by (1), $\left(a_{S}\right)_{L}=\left.\widehat{a}\right|_{L}+a(0)$. For $l \in L, l=l-0$, so $\widehat{a}(l)=a(l)-a(0)$. Thus, $a(l)=\widehat{a}(l)+a(0)$. Then $\left(a_{S}\right)_{L}(l)=\widehat{a}(l)+a(0)=a(l)$. This establishes (2).

Assume that $a \in A(L)$. By $(1), \widehat{a}=\widehat{a}_{S}$. Then $d(a)=\|\widehat{a}\|_{\infty}$ and $d\left(a_{S}\right)=$ $\left\|\widehat{a}_{S}\right\|_{\infty}$ (Theorem 5), so (3) follows from these equalities.

We can now describe the general form of the d-preserving linear bijection raised in the question in $[R R]$ that we mentioned at the beginning of Section 3.

Theorem 9. Let $D: A(K) \rightarrow A(S)$ be a d-preserving linear bijection where $K, S$ are compact convex sets with the former having the property that all the points of $\operatorname{ext}(K)$ are split. [In particular, $K$ could be a Choquet simplex.] We assume, as we may by translation in $A(S)^{*}$, that $0 \in S$. Then there exist a compact convex set $L \subseteq \overline{\mathrm{aco}}(S-S)[S-S$ in the real scalar case], affinely homeomorphic to $K$, such that $\overline{\operatorname{aco}}(S-S)=\overline{\operatorname{aco}}(L-L), 0 \in L$, 
and an affine homeomorphism $\tau: L \rightarrow K$, and $\alpha \in A(L)^{\prime}$ such that for all $a \in A(K)$,

$$
D(a)=c(a \circ \tau)_{S}+\alpha(a), \quad \text { where }|c|=1, \alpha(1) \neq-c .
$$

Proof. We do the proof in the complex scalar case. First we construct $L$. Define $\widetilde{D}: A(K) / \mathbb{C} \rightarrow A(S) / \mathbb{C}$ in the usual way: $\widetilde{D}[a]=[D a]$. Then as seen in the proof of Theorem $6, \widetilde{D}^{*}$ maps $\overline{\operatorname{aco}}(S-S)$ onto $\overline{\operatorname{aco}}(K-K)$. Fix $x_{0} \in \operatorname{ext}(K)$. Define $L=\left(\widetilde{D}^{*}\right)^{-1}\left(K-\left\{x_{0}\right\}\right)$. Then for $x \in L, x \mapsto$ $\widetilde{D}^{*}(x)+x_{0} \in K$, and this map is an affine homeomorphism of $L$ onto $K$. Note $0 \in L$. Observe that $\operatorname{ext}(L)-\operatorname{ext}(L)=\left(\widetilde{D}^{*}\right)^{-1}(\operatorname{ext}(K)-\operatorname{ext}(K))$, and consequently, $\overline{\operatorname{aco}}(L-L)=\left(\widetilde{D}^{*}\right)^{-1}(\overline{\operatorname{aco}}(K-K))=\overline{\operatorname{aco}}(S-S)$. Thus, $S \subseteq S-S \subseteq \overline{\operatorname{aco}}(S-S), 0 \in S$, and $L \subseteq L-L \subseteq \overline{\operatorname{aco}}(L-L), 0 \in L$. Therefore Proposition 8 applies.

Now for $a \in A(K), D(a) \in A(S)$ and $(D(a))_{L} \in A(L)$. By Proposition 8, $a \mapsto(D(a))_{L}$ is a d-preserving linear bijection of $A(K)$ onto $A(L)$. Applying [RR, Theorem 1], we have $(D(a))_{L}=c(a \circ \tau)+\alpha(a)$, where $|c|=1, \alpha \in A(L)^{\prime}$, $\tau: L \rightarrow K$ is an affine homeomorphism, and $\alpha(1) \neq-c$. Using Proposition 8 again, we have

$$
D(a)=\left((D(a))_{L}\right)_{S}=(c(a \circ \tau)+\alpha(a))_{S}=c(a \circ \tau)_{S}+\alpha(a) .
$$

5. A geometric problem involving $K-K, K$ a simplex. Let $K$ be a simplex. We assume that $K$ is embedded in $A(K)^{*}$ as the base of a cone $\widetilde{K}$ which generates $A(K)^{*}$; see [P, p. 59]. It is important to keep in mind that distances between points will be computed in the dual norm on $A(K)^{*}$.

First, let $K$ be the simplex, $K=\operatorname{co}\left\{x_{1}, x_{2}, x_{3}\right\}$. A simple observation using the linear independence of the vectors $x_{1}-x_{2}$ and $x_{2}-x_{3}$ is that

$$
K-K=\left\{\alpha\left(x_{1}-x_{2}\right)+\beta\left(x_{2}-x_{3}\right): \alpha, \beta \in \mathbb{R},|\alpha|+|\alpha-\beta|+|\beta| \leq 2\right\} .
$$

Also, representation of points in $K-K$ is unique. These remarks will be useful in what follows.

The problem is to find polytopes $S$ with the property that $K-K=S-S$.

We may assume by translating that $S \subseteq \widetilde{K}$. Also, we assume that

$$
S=\operatorname{co}\left\{s_{1}, \widetilde{s}_{1}, s_{2}, \widetilde{s}_{2}, s_{3}, \widetilde{s}_{3}\right\} \subseteq A(K)^{*}
$$

is such that

$$
\begin{aligned}
& s_{1}-\widetilde{s}_{1}=x_{1}-x_{2}\left(=y_{1}, \text { say }\right), \quad s_{2}-\widetilde{s}_{2}=x_{2}-x_{3}=y_{2}, \\
& s_{3}-\widetilde{s}_{3}=x_{1}-x_{3}=y_{3},
\end{aligned}
$$

where $\operatorname{ext}(S)=\left\{s_{1}, \widetilde{s}_{1}, s_{2}, \widetilde{s}_{2}, s_{3}, \widetilde{s}_{3}\right\}$. Therefore, $S-S=K-K$. Note that $S-S$ will have only the extreme points $\pm y_{1}, \pm y_{2}, \pm y_{3}$. 
By the decomposition property of vector lattices $[\mathrm{P}, \mathrm{p} .61]$, one sees easily that:

$$
\begin{array}{rll}
s_{1}=x_{1}+a x, & s_{2}=x_{2}+b y, & s_{3}=x_{1}+c z, \\
\widetilde{s}_{1}=x_{2}+a x, & \widetilde{s}_{2}=x_{3}+b y, & \widetilde{s}_{3}=x_{3}+c z,
\end{array}
$$

where $a, b, c \geq 0, x, y, z \in K$.

We first consider the case where the vectors $s_{1}-\widetilde{s}_{1}, s_{2}-\widetilde{s}_{2}$, and $s_{3}-\widetilde{s}_{3}$ intersect in distinct points $P, P^{\prime}$, and $P^{\prime \prime}$; see Fig. 1 .

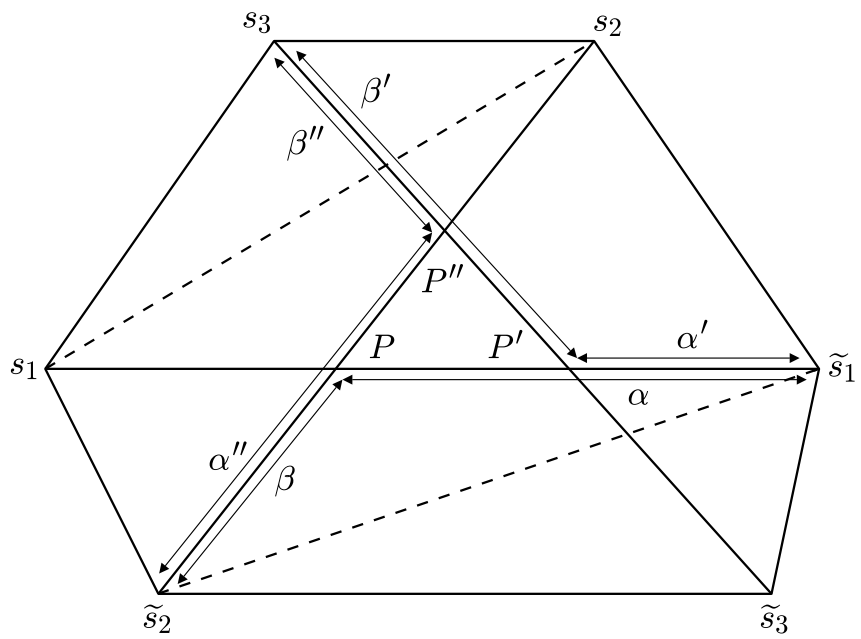

Fig. 1

Consider the point $P$ as a typical case; refer to the quadrilateral $\left\{s_{1}, s_{2}\right.$, $\left.\widetilde{s}_{1}, \widetilde{s}_{2}\right\}$ in Fig. 1. Here

$$
P=\alpha s_{1}+(1-\alpha) \widetilde{s}_{1}=\beta s_{2}+(1-\beta) \widetilde{s}_{2}
$$

(it is assumed that $1 / 2<\alpha<1$ ).

Recall that $\left\|s_{1}-\widetilde{s}_{1}\right\|=\left\|x_{1}-x_{2}\right\|=1+1=2$ by the splittability of extreme points for a simplex. Thus, we have $\alpha=\left\|P-\widetilde{s}_{1}\right\| /\left\|s_{1}-\widetilde{s}_{1}\right\|=$ $\frac{1}{2}\left\|P-\widetilde{s}_{1}\right\|$. Now $P=\alpha\left(s_{1}-\widetilde{s}_{1}\right)+\widetilde{s}_{1}=\beta\left(s_{2}-\widetilde{s}_{2}\right)+\widetilde{s}_{2}$. Also, $\widetilde{s}_{1}-\widetilde{s}_{2}=$ $\beta\left(x_{2}-x_{3}\right)-\alpha\left(x_{1}-x_{2}\right)=(\alpha+\beta) x_{2}-\alpha x_{1}-\beta x_{3}$. Therefore, $\left\|\widetilde{s}_{1}-\widetilde{s}_{2}\right\|=$ $\alpha+\beta+\alpha+\beta=2(\alpha+\beta) \leq 2$, so $\alpha+\beta \leq 1$. Also,

$$
\begin{aligned}
s_{1}-s_{2} & =\left(P-s_{2}\right)+\left(s_{1}-P\right) \\
& =\left[\beta s_{2}+(1-\beta) \widetilde{s}_{2}-s_{2}\right]+\left[s_{1}-\alpha s_{1}-(1-\alpha) \widetilde{s}_{1}\right] \\
& =(1-\beta)\left(\widetilde{s}_{2}-s_{2}\right)+(1-\alpha)\left(s_{1}-\widetilde{s}_{1}\right) \\
& =(1-\beta)\left(x_{3}-x_{2}\right)+(1-\alpha)\left(x_{1}-x_{2}\right) \\
& =(1-\alpha) x_{1}-(2-\alpha-\beta) x_{2}+(1-\beta) x_{3} .
\end{aligned}
$$


This implies that $\left\|s_{1}-s_{2}\right\|=4-2(\alpha+\beta) \leq 2$, so $\alpha+\beta \geq 1$. Thus, $\alpha+\beta=1$. By referring to Fig. 1, we have similarly:

$$
\begin{aligned}
P^{\prime} & =\alpha^{\prime} s_{1}+\left(1-\alpha^{\prime}\right) \widetilde{s}_{1} \\
& =\beta^{\prime} \widetilde{s}_{3}+\left(1-\beta^{\prime}\right) s_{3}, \quad \alpha^{\prime}+\beta^{\prime}=1, \text { where } \alpha^{\prime}=\frac{1}{2}\left\|P^{\prime}-\widetilde{s}_{1}\right\|, \\
P^{\prime \prime} & =\alpha^{\prime \prime} s_{2}+\left(1-\alpha^{\prime \prime}\right) \widetilde{s}_{2} \\
& =\beta^{\prime \prime} \widetilde{s}_{3}+\left(1-\beta^{\prime \prime}\right) s_{3}, \quad \alpha^{\prime \prime}+\beta^{\prime \prime}=1, \text { where } \alpha^{\prime \prime}=\frac{1}{2}\left\|P^{\prime \prime}-\widetilde{s}_{2}\right\| .
\end{aligned}
$$

To find the relations among $\alpha, \beta, \alpha^{\prime}, \beta^{\prime}, \alpha^{\prime \prime}, \beta^{\prime \prime}$, argue as follows:

$$
\begin{aligned}
P-s_{3} & =\left(P-P^{\prime \prime}\right)+\left(P^{\prime \prime}-s_{3}\right)=\left(1-\beta-\beta^{\prime \prime}\right)\left(\widetilde{s}_{2}-s_{2}\right)+\beta^{\prime \prime}\left(\widetilde{s}_{3}-s_{3}\right) \\
& =\left(\alpha-\beta^{\prime \prime}\right)\left(\widetilde{s}_{2}-s_{2}\right)+\beta^{\prime \prime}\left(\widetilde{s}_{3}-s_{3}\right) \\
& =\left(\beta^{\prime \prime}-\alpha\right)\left(x_{2}-x_{3}\right)-\beta^{\prime \prime}\left(x_{1}-x_{3}\right) \\
& =-\beta^{\prime \prime}\left(x_{1}-x_{2}\right)-\alpha\left(x_{2}-x_{3}\right) .
\end{aligned}
$$

Also,

$$
\begin{aligned}
P-s_{3} & =\left(P-P^{\prime}\right)+\left(P^{\prime}-s_{3}\right)=\left(1-\alpha^{\prime}-\beta\right)\left(s_{1}-\widetilde{s}_{1}\right)+\beta^{\prime}\left(\widetilde{s}_{3}-s_{3}\right) \\
& =\left(\beta^{\prime}-\beta\right)\left(x_{1}-x_{2}\right)-\beta^{\prime}\left(x_{1}-x_{3}\right)=-\beta\left(x_{1}-x_{2}\right)-\beta^{\prime}\left(x_{2}-x_{3}\right) .
\end{aligned}
$$

It follows by the uniqueness of representation of points in $K-K$ that $\beta=\beta^{\prime \prime}$ and $\alpha=\beta^{\prime}$. Then $1=\alpha+\beta=\beta^{\prime}+\beta^{\prime \prime}$, so $\alpha^{\prime \prime}=\beta^{\prime}$ and $\alpha^{\prime}=\beta^{\prime \prime}$. Therefore, $\alpha=\beta^{\prime}=\alpha^{\prime \prime}$ and $\beta=\alpha^{\prime}=\beta^{\prime \prime}$.

Note that now the various distances can be computed. For example,

$$
s_{2}-\widetilde{s}_{1}=\left(s_{2}-P\right)+\left(P-\widetilde{s}_{1}\right)=\alpha\left(s_{2}-\widetilde{s}_{2}\right)+\alpha\left(s_{1}-\widetilde{s}_{1}\right)=\alpha\left(s_{3}-\widetilde{s}_{3}\right) .
$$

Therefore, $\left\|s_{2}-\widetilde{s}_{1}\right\|=2 \alpha$. This also shows that $s_{2}-\widetilde{s}_{1}$ is not extreme in $S-S$. The same kind of argument shows that $\left\{s_{i}-\widetilde{s}_{i}\right\}_{i=1,2,3}$ are the only extreme points in $S-S$.

Returning to equation (\$), we can explicitly write down the form of $s_{1}, \widetilde{s}_{1}, s_{2}, \widetilde{s}_{2}, s_{3}, \widetilde{s}_{3}$ as follows (from Fig. 1):

$$
\begin{aligned}
P & =\alpha s_{1}+\beta \widetilde{s}_{1}=\alpha\left(x_{1}+a x\right)+\beta\left(x_{2}+a x\right)=\alpha x_{1}+\beta x_{2}+a x \\
& =\alpha \widetilde{s}_{2}+\beta s_{2}=\alpha\left(x_{3}+b y\right)+\beta\left(x_{2}+b y\right)=\alpha x_{3}+\beta x_{2}+b y .
\end{aligned}
$$

This implies $\alpha\left(x_{1}-x_{3}\right)=b y-a x$, so $b=a$ and $b y=\alpha\left(x_{1}-x_{3}\right)+a x$. Similarly, $c=a$ and $c z=\alpha\left(x_{2}-x_{3}\right)+a x$. Thus,

$$
\begin{array}{ll}
s_{1}=x_{1}+a x, & \widetilde{s}_{1}=x_{2}+a x, \\
s_{2}=x_{2}+\alpha\left(x_{1}-x_{3}\right)+a x, & \widetilde{s}_{2}=x_{3}+\alpha\left(x_{1}-x_{3}\right)+a x, \\
s_{3}=x_{1}+\alpha\left(x_{2}-x_{3}\right)+a x, & \widetilde{s}_{3}=x_{3}+\alpha\left(x_{2}-x_{3}\right)+a x .
\end{array}
$$

REMARKs. (1) The above is the general solution when $\alpha>1 / 2$. When $\alpha \rightarrow 1 / 2$, we check that $P_{0}=\frac{1}{2}\left(s_{1}+\widetilde{s}_{1}\right)=\frac{1}{2}\left(s_{2}+\widetilde{s_{2}}\right)=\frac{1}{2}\left(s_{3}+\widetilde{s}_{3}\right)=$ $\frac{1}{2}\left(x_{1}+x_{2}\right)+a x$, and we get the symmetric solution $S=\frac{1}{2}(K-K)+P_{0}$. 
(2) When $\alpha \rightarrow 1$, we get

$$
\begin{array}{lll}
s_{1}=x_{1}+a x, & s_{2}=x_{1}+x_{2}-x_{3}+a x, & s_{3}=x_{1}+x_{2}-x_{3}+a x, \\
\widetilde{s}_{1}=x_{2}+a x, & \widetilde{s}_{2}=x_{1}+a x, & \widetilde{s}_{3}=x_{2}+a x .
\end{array}
$$

Hence, $s_{1}=\widetilde{s}_{2}, \widetilde{s}_{1}=\widetilde{s}_{3}$ and $s_{2}=s_{3}$, and we get the simplex $\operatorname{co}\left\{x_{1}, x_{1}+\right.$ $\left.x_{2}-x_{3}, x_{2}\right\}$. Translating by $x_{3}$, we have the simplex co $\left\{x_{1}+x_{3}, x_{1}+x_{2}\right.$, $\left.x_{2}+x_{3}\right\}$, and the latter is obtained by translating $\operatorname{co}\left\{-x_{1},-x_{2},-x_{3}\right\}$ by $x_{1}+x_{2}+x_{3}$.

(3) "Uniqueness" of solutions. To show that there cannot be points in $S$ other than those specified, take $s$ as a typical point outside $\operatorname{co}\left\{s_{1}, \widetilde{s_{1}}, s_{2}, \widetilde{s}_{2}\right.$, $\left.s_{3}, \widetilde{s}_{3}\right\}$ of the form

$$
s=\widetilde{s}_{1}+t_{0}\left(\widetilde{s}_{1}-s_{1}\right)+t\left(s_{2}-\widetilde{s}_{1}\right), \quad t_{0}>0,0<t<1 .
$$

Then from (\%) we obtain $s=x_{2}+t_{0}\left(x_{2}-x_{1}\right)+t \alpha\left(x_{1}-x_{3}\right)+a x$ and $\widetilde{s}_{2}=$ $x_{3}+\alpha\left(x_{1}-x_{3}\right)+a x$. Hence,

$$
\begin{aligned}
s-\widetilde{s}_{2} & =x_{2}-x_{3}+\alpha(t-1)\left(x_{1}-x_{3}\right)-t_{0}\left(x_{1}-x_{2}\right) \\
& =-\left[\alpha(1-t)+t_{0}\right]\left(x_{1}-x_{2}\right)+[1-\alpha(1-t)]\left(x_{2}-x_{3}\right) \\
& =a\left(x_{1}-x_{2}\right)+b\left(x_{2}-x_{3}\right) .
\end{aligned}
$$

Then $|a|+|b|+|a-b|=\alpha(1-t)+t_{0}+1-\alpha(1-t)+\left|1+t_{0}\right|=2\left(1+t_{0}\right)>2$. It follows that $s-\widetilde{s}_{2} \notin K-K$.

The problem in $\mathbb{R}^{3}$. Consider the simplex $K=\operatorname{co}\left\{x_{1}, x_{2}, x_{3}, x_{4}\right\}$ in $\mathbb{R}^{3}$. Let $S=\left\{s_{1}, \widetilde{s}_{1}, s_{2}, \widetilde{s}_{2}, \ldots, s_{6}, \widetilde{s}_{6}\right\}$. Again, we want to find conditions under which $S-S=K-K$. For this purpose we use the equations in (\%). By $(\%)$, for $\operatorname{co}\left\{x_{1}, x_{2}, x_{3}\right\}$, with $1 / 2<\alpha_{1}<1$, we have:

$$
\begin{array}{ll}
s_{1}=x_{1}+a x, & \widetilde{s}_{1}=x_{2}+a x, \\
s_{2}=x_{2}+\alpha_{1}\left(x_{1}-x_{3}\right)+a x, & \widetilde{s}_{2}=x_{3}+\alpha_{1}\left(x_{1}-x_{3}\right)+a x, \\
s_{3}=x_{1}+\alpha_{1}\left(x_{2}-x_{3}\right)+a x, & \widetilde{s}_{3}=x_{3}+\alpha_{1}\left(x_{2}-x_{3}\right)+a x .
\end{array}
$$

Similarly, for $\operatorname{co}\left\{x_{1}, x_{2}, x_{4}\right\}$, with $1 / 2<\alpha_{1}<1, a_{1} \bar{x}$, we have:

$$
\begin{array}{ll}
s_{1}=x_{1}+a_{1} \bar{x}, & \widetilde{s}_{1}=x_{2}+a_{1} \bar{x} \\
s_{5}=x_{2}+\alpha_{2}\left(x_{1}-x_{4}\right)+a_{1} \bar{x}, & \widetilde{s}_{5}=x_{4}+\alpha_{2}\left(x_{1}-x_{4}\right)+a_{1} \bar{x} \\
s_{6}=x_{1}+\alpha_{2}\left(x_{2}-x_{4}\right)+a_{1} \bar{x}, & \widetilde{s}_{6}=x_{4}+\alpha_{2}\left(x_{2}-x_{4}\right)+a_{1} \bar{x} .
\end{array}
$$

Note that from the first two equations in the systems $(\mathrm{A})$ and $(\mathrm{B})$, it is clear that $a x=a_{1} \bar{x}$. For $\operatorname{co}\left\{x_{1}, x_{3}, x_{4}\right\}$, with $\frac{1}{2}<\alpha_{3}<1, a_{2} \overline{\bar{x}}$, we have:

$$
\begin{array}{ll}
s_{3}=x_{1}+\alpha_{3}\left(x_{4}-x_{1}\right)+a_{2} \overline{\bar{x}}, & \widetilde{s}_{3}=x_{3}+\alpha_{3}\left(x_{4}-x_{1}\right)+a_{2} \overline{\bar{x}} \\
s_{4}=x_{4}+a_{2} \overline{\bar{x}} & \widetilde{s}_{4}=x_{3}+a_{2} \overline{\bar{x}} \\
s_{6}=x_{1}+\alpha_{3}\left(x_{3}-x_{1}\right)+a_{2} \overline{\bar{x}}, & \widetilde{s}_{6}=x_{4}+\alpha_{3}\left(x_{3}-x_{1}\right)+a_{2} \overline{\bar{x}}
\end{array}
$$


Equating $s_{6}$ from (B) and (C), we have

$$
s_{6}=x_{1}+\alpha_{2}\left(x_{2}-x_{4}\right)+a_{1} \bar{x}=s_{6}=x_{1}+\alpha_{3}\left(x_{3}-x_{1}\right)+a_{2} \overline{\bar{x}},
$$

and this implies $a=a_{2}$ and $a_{2} \overline{\bar{x}}=\alpha_{2}\left(x_{2}-x_{4}\right)+\alpha_{3}\left(x_{1}-x_{3}\right)+a x$. Then using the fifth equation in $(\mathrm{C})$, we obtain

$s_{3}=x_{1}+\alpha_{3}\left(x_{4}-x_{1}\right)+\alpha_{2}\left(x_{2}-x_{4}\right)+\alpha_{3}\left(x_{1}-x_{3}\right)+a x=x_{1}+\alpha_{1}\left(x_{2}-x_{3}\right)+a x$ (by the fifth equation in (A)). It follows that

$$
\alpha_{3}\left(x_{4}-x_{1}\right)+\alpha_{2}\left(x_{2}-x_{4}\right)+\alpha_{3}\left(x_{1}-x_{3}\right)=\alpha_{1}\left(x_{2}-x_{3}\right),
$$

or

$\alpha_{3}\left(x_{4}-x_{1}\right)-\alpha_{2}\left(x_{4}-x_{2}\right)+\alpha_{3}\left(x_{1}-x_{4}+x_{4}-x_{3}\right)=\alpha_{1}\left(x_{2}-x_{4}+x_{4}-x_{3}\right)$,

or

$$
\left(\alpha_{1}-\alpha_{2}\right)\left(x_{2}-x_{4}\right)+\left(\alpha_{1}-\alpha_{3}\right)\left(x_{4}-x_{3}\right)=0 .
$$

By the linear independence of the vectors $\left\{x_{1}-x_{4}, x_{2}-x_{4}, x_{3}-x_{4}\right\}$, we must have $\alpha_{1}-\alpha_{2}=0=\alpha_{1}-\alpha_{3}$, which implies $\alpha_{1}=\alpha_{2}=\alpha_{3}$ (= $\alpha$, say), and the solution can now be written

$$
\begin{array}{ll}
s_{1}=x_{1}+a x, & \widetilde{s}_{1}=x_{2}+a x, \\
s_{2}=x_{2}+\alpha\left(x_{1}-x_{3}\right)+a x, & \widetilde{s}_{2}=x_{3}+\alpha\left(x_{1}-x_{3}\right)+a x, \\
s_{3}=x_{1}+\alpha\left(x_{2}-x_{3}\right)+a x, & \widetilde{s}_{3}=x_{3}+\alpha\left(x_{2}-x_{3}\right)+a x, \\
s_{4}=x_{4}+a\left(x_{1}+x_{2}-x_{3}-x_{4}\right)+a x, & \widetilde{s}_{4}=x_{3}+a\left(x_{1}+x_{2}-x_{3}-x_{4}\right)+a x, \\
s_{5}=x_{2}+\alpha\left(x_{1}-x_{4}\right)+a x, & \widetilde{s}_{5}=x_{4}+\alpha\left(x_{1}-x_{4}\right)+a x, \\
s_{6}=x_{1}+\alpha\left(x_{2}-x_{4}\right)+a x, & \widetilde{s}_{6}=x_{4}+\alpha\left(x_{2}-x_{4}\right)+a x .
\end{array}
$$

The form of this solution simplifies if one translates by $\alpha\left(x_{3}+x_{4}\right)$ :

$$
\begin{array}{ll}
s_{1}=x_{1}+\alpha\left(x_{3}+x_{4}\right)+a x, & \widetilde{s}_{1}=x_{2}+\alpha\left(x_{3}+x_{4}\right)+a x, \\
s_{2}=x_{2}+\alpha\left(x_{1}+x_{4}\right)+a x, & \widetilde{s}_{2}=x_{3}+\alpha\left(x_{1}+x_{4}\right)+a x, \\
s_{3}=x_{1}+\alpha\left(x_{2}+x_{4}\right)+a x, & \widetilde{s}_{3}=x_{3}+\alpha\left(x_{2}+x_{4}\right)+a x, \\
s_{4}=x_{4}+a\left(x_{1}+x_{2}\right)+a x, & \widetilde{s}_{4}=x_{3}+a\left(x_{1}+x_{2}\right)+a x, \\
s_{5}=x_{2}+\alpha\left(x_{1}+x_{3}\right)+a x, & \widetilde{s}_{5}=x_{4}+\alpha\left(x_{1}+x_{3}\right)+a x, \\
s_{6}=x_{1}+\alpha\left(x_{2}+x_{3}\right)+a x, & \widetilde{s}_{6}=x_{4}+\alpha\left(x_{2}+x_{3}\right)+a x .
\end{array}
$$

As before, one checks easily that $s_{i}-s_{j}, s_{i}-\widetilde{s}_{j}$, and $\widetilde{s}_{i}-\widetilde{s}_{j}$ are not extreme for $i \neq j$.

Remarks. (1) The points $\left\{s_{i}-\widetilde{s}_{i}\right\}_{i=1}^{6}$ are the extreme points of $S-S$. It is a curious fact that the points in this set are equidistant from $x_{1}+x_{2}+$ $x_{3}+x_{4}$ [in the $A(K)^{*}$ metric].

(2) It is more or less clear that the set $S$ is non-symmetric, but here is a formal proof of this fact: If $P_{0}$ were the centre of symmetry of $S$, then for each extreme point (say, $s_{1}$ ), there exists $t_{1} \in S$ such that $\left(s_{1}+t_{1}\right) / 2=P_{0}$. 
This implies $s_{1}-P_{0}=P_{0}-t_{1}$. Similarly, $\widetilde{s}_{1}-P_{0}=P_{0}-t_{2}$ for some $t_{2} \in S$. Then we must have $s_{1}-\widetilde{s}_{1}=t_{2}-t_{1}$, and so $s_{1}=t_{2}$ and $\widetilde{s}_{1}=t_{1}$ (by the uniqueness of the expression of an extreme point in $S-S$ ). Thus, $P_{0}=\left(s_{1}+\widetilde{s}_{1}\right) / 2=\frac{1}{2}\left(x_{1}+x_{2}\right)+a x$. But $\left(s_{2}+\widetilde{s}_{2}\right) / 2$ is something different, which is a contradiction (unless $\alpha=1 / 2$ ).

(3) $\alpha \rightarrow 1 / 2$ gives the symmetric solution as before with centre of symmetry $\frac{1}{2}\left(x_{1}+x_{2}\right)$.

(4) $\alpha \rightarrow 1$ gives a translate of $K$ as a solution.

Uniqueness. Since the solution for the simplex $K=\operatorname{co}\left\{x_{1}, x_{2}, x_{3}, x_{4}\right\}$ in $\mathbb{R}^{3}$ was obtained by solving for each face, the solution should therefore be unique, modulo translation and the ordering of the points $s_{1}, \widetilde{s}_{1}, s_{2}, \widetilde{s}_{2}, \ldots$, $s_{6}, \widetilde{s}_{6}$.

It is now apparent what the solution for $K=\operatorname{co}\left\{x_{1}, x_{2}, x_{3}, x_{4}, x_{5}\right\}$ in $\mathbb{R}^{4}$ will be like:

$$
\begin{aligned}
\left\{x_{1}, x_{2}\right\} \rightarrow & s_{1}=x_{1}+\alpha\left(x_{3}+x_{4}+x_{5}\right)+a x, \\
& \widetilde{s}_{1}=x_{2}+\alpha\left(x_{3}+x_{4}+x_{5}\right)+a x, \\
\left\{x_{1}, x_{3}\right\} \rightarrow & s_{2}=x_{1}+\alpha\left(x_{1}+x_{4}+x_{5}\right)+a x, \\
& \widetilde{s}_{2}=x_{3}+\alpha\left(x_{1}+x_{4}+x_{5}\right)+a x, \\
\left\{x_{1}, x_{4}\right\} \rightarrow & \\
\left\{x_{1}, x_{5}\right\} \rightarrow & \\
\vdots & \\
\left\{x_{4}, x_{5}\right\} \rightarrow & s_{10}=x_{4}+\alpha\left(x_{1}+x_{2}+x_{3}\right)+a x, \\
& \widetilde{s}_{10}=x_{5}+\alpha\left(x_{1}+x_{2}+x_{3}\right)+a x .
\end{aligned}
$$

It is now clear that a solution $S$ for a simplex $K=\operatorname{co}\left\{x_{1}, x_{2}, \ldots, x_{n+1}\right\}$ in $\mathbb{R}^{n}$ can be written down by inspection.

This paper was written while the first author was a Visiting Scientist at ISI-Calcutta. He thanks ISI-Calcutta for this opportunity, and he especially thanks his colleagues at ISI for their very warm hospitality.

We would like to thank Professor T. S. S. R. K. Rao for some helpful correspondence on the subject of this paper. Also, we thank the referee for his comments.

\section{References}

[A] E. Alfsen, Compact Convex Sets and Boundary Integrals, Springer, Berlin, 1971.

[AE] L. Asimow and A. J. Ellis, Convexity Theory and Its Applications in Functional Analysis, London Math. Soc. Monographs 16, Academic Press, 1980.

[B] S. Banach, Théorie des Opérations Linéaires, Monografje Mat. 1, Warszawa, 1932. 
[D] J. Dugundji, Topology, Allyn and Bacon, Boston, 1966.

[DS] N. Dunford and J. T. Schwartz, Linear Operators, Vol. I, Interscience, New York, 1958.

[GU] F. González and V. Uspenskij, On homomorphisms of groups of integer-valued functions, Extracta Math. 14 (1999), 19-29.

[GM] M. Györy and L. Molnár, Diameter preserving linear bijections of $C(X)$, Arch. Math. (Basel) 71 (1998), 301-310.

[LT] D. Lay and A. Taylor, Functional Analysis, Wiley, 1990.

[MU] S. Mazur et S. Ulam, Sur les transformations isométriques d'espaces vectoriels normés, C. R. Acad. Sci. Paris 194 (1932), 946-948.

[P] R. R. Phelps, Lectures on Choquet's Theorem, Van Nostrand, Princeton, 1965.

$[\mathrm{RR}] \quad$ T. S. S. R. K. Rao and A. K. Roy, Diameter-preserving linear bijections of function spaces, J. Austral. Math. Soc. Ser. A 70 (2001), 323-335.

[R] W. Rudin, Functional Analysis, McGraw-Hill, 1991.

[S] F. C. Sanchez, Diameter preserving linear maps and isometries (II), Proc. Indian Acad. Sci. Math. Sci. 110 (2000), 205-211.

Department of Mathematics

University of Oregon

Eugene, OR 97403, U.S.A.

E-mail: barnes@math.uoregon.edu
Indian Statistical Institute-Calcutta

Statistics and Mathematics Unit

203 B. T. Road

Calcutta 700 035, India

E-mail: ashoke@isical.ac.in

Received August 20, 2001

Revised version April 15, 2002 